\title{
Glomus fasciculatum, a dominant arbuscular mycorrhizal species in the rhizosphere soils of Setaria italica in Mahabubnagar district of Telangana state, India
}

\author{
Hari Prasad Kante ${ }^{* 1}$, Laxmi Kanth Mhadgula ${ }^{2}$ \\ ${ }^{*}$ Assistant Professor, Department of Botany, S.V. Government Arts \& Science College, Palem, Telangana, India. \\ ${ }^{2}$ Post Graduate in Botany, Government Degree \& PG College, Wanaparthy, Telangana, India.
}

\begin{abstract}
The arbuscular mycorrhizal (AM) fungal association in the rhizosphere soils of Setaria italica (L.) P. Beauv. from 8 Mandals of Mahabubnagar district, Telangana state, India, were studied. A significant number of AM fungi were identified in the present study, i.e., 53. Among the 53 AM fungi, Glomus was represented by 23 species, Acaulospora with 11, Sclerocystis with 6, Scutellospora with 5, Gigaspora with 4, and Funneliformis with 2, Ambispora and Archaeospora with 1 species. Glomus fasciculatum was the dominant species among the Glomus genera isolated from the rhizosphere soil samples of 34 villages in Mahabub Nagar district of Telangana State. The AM fungi spore density ranged from 74 to 270 per $100 \mathrm{gm}$ soil (average 145), while the root colonization ranged from $50.67 \%$ to $93.33 \%$ (average $75.71 \%$ ). This study provides valuable information on $\mathrm{AM}$ fungal association in Setaria italica.
\end{abstract}

Keywords : Glomus Fasciculatum, Setaria Italica, Arbuscular Mycorrhizal Fungi, AM Fungal Spores, Root Colonization.

\section{INTRODUCTION}

Arbuscular Mycorrhizal fungi play a significant role in the Plant growth and metabolism. Foxtail millets (Setaria italica) are an important group of plants predominantly cultivated and consumed by people in Asia and Africa. The seeds of foxtail millets are rich in essential nutrients including calcium, magnesium and iron. Foxtail millets are used as a major source of food for millions of people. Foxtail millet (Setaria italica), probably first cultivated some 8,000 years ago in China, is widely grown as a grain crop not only in the semi-arid regions of Asia (India, China and Japan) but also in Southern Europe, and is becoming an increasingly important forage crop in the Americas, Australia and North Africa [1].

\section{METHODS AND MATERIAL}

Geographical details of the Study area:

The Mahabubnagar district is located between $15^{\circ} 55^{\prime}$ and $17^{\circ} 29^{\prime} \mathrm{N}$ latitudes and between $77^{\circ} 15^{\prime}$ and $79^{\circ} 15^{\prime}$ E longitudes in Telangana state, India. To evaluate the diversity of AM fungi in rhizosphere soils and its association with roots of Setaria italica, the root samples and rhizosphere soils were collected from 8 different mandals (Alampur, Kodair, Kollapur, Ieeja, Itikyal, Manopad, Veepangandla and Waddepally). 
The soil type of this area is sandy loam soils and clay loam soils.

MAP.1 : The rhizosphere soil sample collection points of 34 villages from 8 mandals in Mahabubnagar district, Telangana, India. ( $1=$ Alampur; $2=$ Kodair; $3=$ Kollapur; 4=Ieeja; 5= Itikyal; 6= Manopad; 7= Veepangandla; 8= Waddepally)
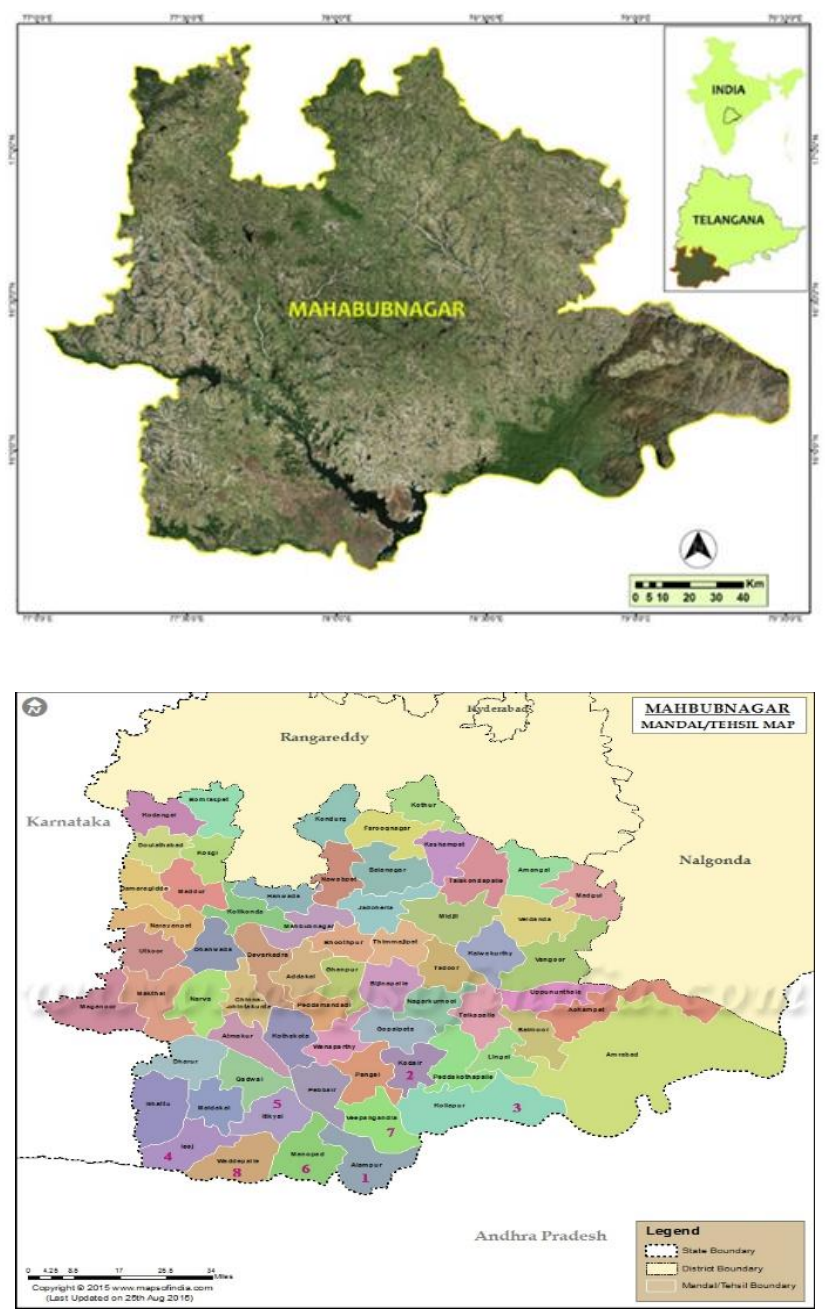

\section{Collection of root and soil samples:}

The mixtures of roots and rhizosphere soils of Setaria italica were collected from a depth of ca.5-10 cm. Three healthy plants were selected from each field, root and soil samples were collected and subsequently placed in plastic bags and transferred to the laboratory. In the laboratory, the rhizosphere soil was air dried and stored in a refrigerator at $4{ }^{\circ} \mathrm{C}$ until processing. The root samples were washed with distilled water to removed attached soil particles, cut into $1 \mathrm{~cm}$ segments and fixed in FAA solution (formalin, acetic acid and alcohol).

Isolation, identification and quantification of $\mathrm{AM}$ Fungi spore:

Spores and sporocarps of AM fungi were isolated by using the wet sieving and decanting method [2]. AMF spore identification and their morphological characters were determined and analyzed qualitatively by using manual of Schenck and Perez [3] and the website of the

International collection of vesicular and AM fungi (http://invam.wvu.edu/; http://www.zor.zut.edu.pl/Glomeromycota/index.htm l).

\section{Quantification of AM fungi root colonization:}

The root samples were gently washed with distilled water to remove attached soil particles and free of FAA. The root samples cut into approximately $1 \mathrm{~cm}$ long segments, cleared by boiling in $10 \% \mathrm{KOH}$ and the boiling time varied depending on the colour and thickness of the roots [4]. Cleared root segments were acidified with $5 \mathrm{~N} \mathrm{HCl}$ and stained with $0.05 \%$ trypan blue in lactophenol [5]. AM fungal colonization was quantified by the glass side method, in which 50 randomly selected $1 \mathrm{~cm}$ long root segments were examined microscopically [6]. The root colonization percentage was calculated by the following formulae:

$=$ No. of infected root fragments $\times 100$

No. of total root fragments

A significant number of AM fungi were identified in the present study, i.e., 53. among the 53 AM fungi, Glomus was represented by 23 species, Acaulospora with 11, Sclerocystis with 6, Scutellospora with 5, 
Gigaspora with 4, and Funneliformis with 2, Ambispora and Archaeospora with 1 species. Glomus fasciculatum was the dominant species among the Glomus genera isolated from the rhizosphere soil samples of 34 villages in Mahabub Nagar district of Telangana State. The AM fungi spore density ranged (Table.1) from 74 to 270 per 100 gm soil (average 145), while the root colonization ranged from $50.67 \%$ to 93.33\% (average 75.71\%). This study provides valuable information on AM fungal association in Setaria italica.

The AM fungal association with Setaria italica in 8 Mandals of Mahabubnagar District investigated in the present study. From the research, we could conclude that the biodiversity of AM fungi was abundant, though Glomus fasciculatum (Fig. 1\&2) was the dominant species found in all 34 samples. The AM fungal spore density and root colonization varied markedly among 8 Mandals. Considering the potential application of AM fungi Glomus fasciculatum on Setaria italica, it seems that more attention should be paid to the predominant $\mathrm{AM}$ fungi during the process of their cultivation, especially mycorrhizal performance i.e., improving growth, increasing secondary metabolite production.

\section{Glomus fasciculatum (Thaxt.) Gerd. \& Trappe emend. C. Walker \& Koske.}

Spores single in the soil or in aggregates with 2-20 spores, lacking a peridium. Pale yellow colour, globose to subglobose; (50-) 105 (-130) $\mu \mathrm{m}$ diam; with one subtending hypha. Spores composed of one wall with three layers (swl1-3). Subtending hypha, pale yellow; straight or slightly curved; cylindrical; (5.0)12.5(-16.0) $\mu \mathrm{m}$ wide at the spore base

\section{CONCLUSION}

In the present study, Glomus fasciculatum was the dominant species (Table. 2) among the Glomus genera isolated from the rhizosphere soil samples of 34 villages in Mahabubnagar district of Telangana State. Most of the soil samples belongs to the sandy loam soils. The morphological characters of Glomus fasciculatum were well fitted. All the 34 rhizosphere soil samples of Setaria italica associated with Glomus fasciculatum. The Foxtail millet inoculated with AM fungus Glomus fasciculatum showed increased value for growth, biomass and phosphorus uptake over the remaining treatments [7]. Mycorrhizal inoculation helped in enhancing the biomass of plant, per cent mycorrhizal colonization and spore number due to increased uptake of mineral nutrients.

\section{ACKNOWLEDGEMENT}

I would like to thank Southern Eastern Regional Office, Hyderabad for sanctioning minor research project, which has helped me complete the research work and also strengthen the lab facilities in Government Degree College.

\section{REFERENCES}

[1]. Bennetzen JL, Schmutz J, Wang H, Percifield R, Hawkins J, et al. 2012. Reference genome sequence of the model plant Setaria. Nature Biotechnology 30: 555-561.

[2]. Gerdemann J.W. and Nicolson T.H. 1963. Spores of mycorrhizal Endogone species extracted from soil by Wet-Sieving and Decanting. Trans.Br. Mycol. Soc. 46: 235-244.

[3]. Schenck N.C. and Perez Y. 1990. Manual for the Identification of VA Mycorrhizal Fungi. Synergistic Publications, Gainesville, Florida, USA. pp 283.

[4]. Koske and Gemma, 1989. A modified procedure for staining roots to detect VA mycorrhizas. Mycological Research. Volume 92, Issue 4, June 1989, Pages 486-488. 
[5]. Phillips J. and Hayman D.S. 1970. Improved procedure for clearing roots and staining parasitic and vesicular mycorrhizal fungi for rapid assessment of infection. Trans. Br. Mycol. Soc. 55: $158-161$.

[6]. Giovannetti, M., \& Mosse, B., 1980. An Evaluation of Techniques for Measuring Vesicular Arbuscular Mycorrhizal Infection in Roots. New Phytologist, 84, 489-500. http://dx.doi.org/10.1111/j.14698137.1980.tb045 56.x.

[7]. Channabasava A. and H. C. Lakshman, 2012.Mycorrhization effect on biomass of four rare millets of North Karnataka (INDIA)

[8]. International Journal of Agricultural Sciences ISSN: 2167-0447 Vol. 2 (8), pp. 235-239.
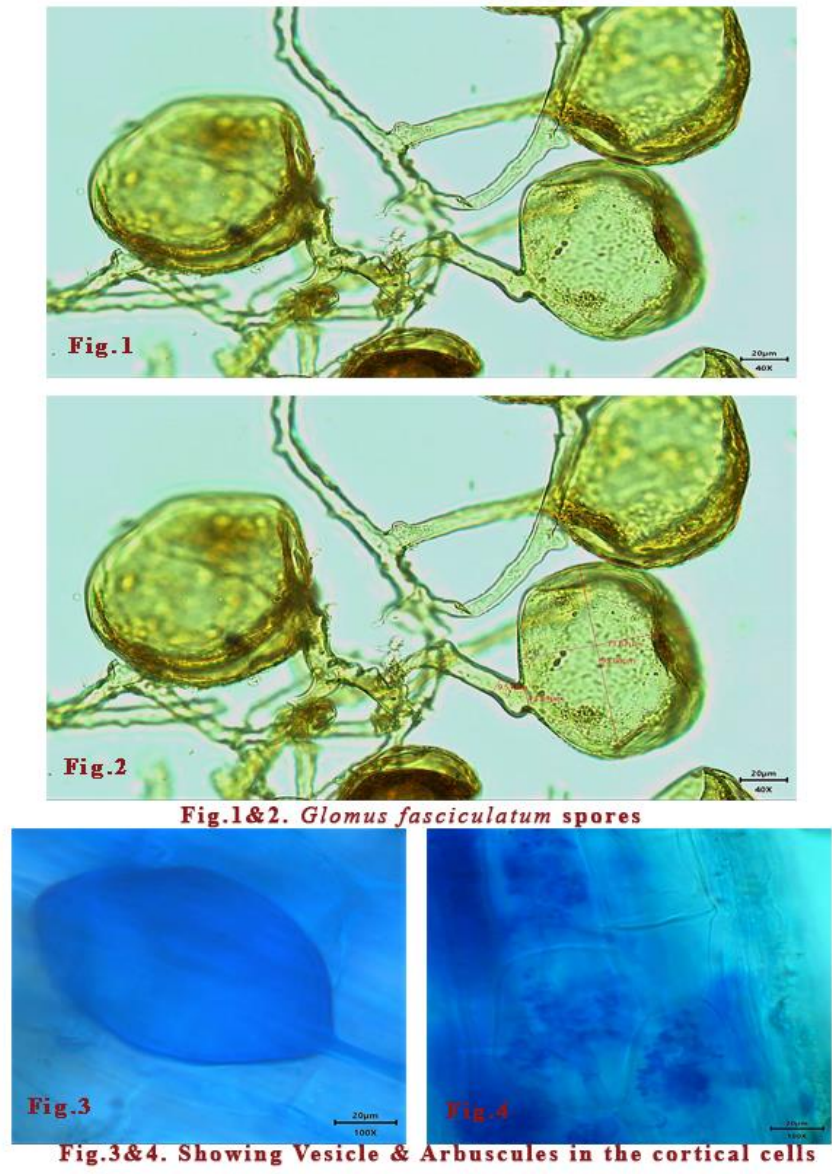

of Setaria Italica

Table 1 : AM fungal spore count and percentage of root colonization in Setaria italica from 8 mandals of 34 villages in Mahabubnagar district

\begin{tabular}{|c|l|l|c|c|}
\hline SL.NO & \multicolumn{1}{|c|}{ NAME OF THE VILLAGE } & \multicolumn{1}{c|}{ SOIL TYPE } & Root colonization \% & No.of Spores/100gm of Soil \\
\hline $\mathbf{1}$ & ALAMPUR & Black & 56 & 113 \\
\hline $\mathbf{2}$ & BHEEMAVARAM & Sandy loam & 84 & 270 \\
\hline $\mathbf{3}$ & KONERU & Sandy loam & 80 & 200 \\
\hline $\mathbf{4}$ & KYATUR & Sandy loam & 93.33 & 120 \\
\hline $\mathbf{5}$ & LIGANVAI & Sandy loam & 88 & 136 \\
\hline $\mathbf{6}$ & MARAMUNAGALA & Black & 66.67 & 111 \\
\hline $\mathbf{7}$ & UTKUR & Sandy loam & 82.67 & 129 \\
\hline $\mathbf{8}$ & ETTAM & Sandy loam & 76 & 86 \\
\hline $\mathbf{9}$ & NARSAIPALLE & Sandy loam & 72 & 106 \\
\hline $\mathbf{1 0}$ & CHUKKAIPALLE & Sandy loam & 72 & 129 \\
\hline $\mathbf{1 1}$ & KOLLAPUR & Sandy loam & 82.67 & 74 \\
\hline $\mathbf{1 2}$ & KUDIKILLA & Sandy loam & 50.67 & 239 \\
\hline $\mathbf{1 3}$ & MADHAVASWAMY NAGAR & Sandy loam & 77.33 & 85 \\
\hline $\mathbf{1 4}$ & MALLESWARAM & Sandy loam & 74.67 & 110 \\
\hline $\mathbf{1 5}$ & MANCHALAKATTA & Sandy loam & 78.67 & 128 \\
\hline $\mathbf{1 6}$ & PENTLAVELLY & Black & 89.33 & \\
\hline
\end{tabular}




\begin{tabular}{|l|l|l|c|c|}
$\mathbf{1 7}$ & RAMAPUR & Sandy loam & 90.67 & 111 \\
\hline $\mathbf{1 8}$ & SOMASHILA & Sandy loam & 62.67 & 121 \\
\hline $\mathbf{1 9}$ & YANGAM PALLY & Sandy loam & 72 & 208 \\
\hline $\mathbf{2 0}$ & YELLUR & Sandy loam & 78.67 & 206 \\
\hline $\mathbf{2 1}$ & IEEJA & Sandy loam & 81.33 & 97 \\
\hline $\mathbf{2 2}$ & TUPPADITALLA & Black & 74.67 & 86 \\
\hline $\mathbf{2 3}$ & VENKATAPUR & Sandy loam & 73.33 & 75 \\
\hline $\mathbf{2 4}$ & YAPADINNE & Sandy loam & 76 & 239 \\
\hline $\mathbf{2 5}$ & ITIKYAL & Black & 85.33 & 94 \\
\hline $\mathbf{2 6}$ & SATERLA & Black & 88 & 212 \\
\hline $\mathbf{2 7}$ & UDANDAPUR & Black & 53.33 & 114 \\
\hline $\mathbf{2 8}$ & KALUGOTLA & Black & 82.67 & 134 \\
\hline $\mathbf{2 9}$ & POTULAPADU & Black & 80 & 97 \\
\hline $\mathbf{3 0}$ & KONDURU & Sandy loam & 60 & 224 \\
\hline $\mathbf{3 1}$ & \multirow{2}{*}{ RAJOLI } & Black & 73 & 160 \\
\hline $\mathbf{3 2}$ & RAMAPURAM & Black & 72 & 137 \\
\hline $\mathbf{3 3}$ & TIMMAJIPALLE & Sandy loam & 70.67 & 237 \\
\hline $\mathbf{3 4}$ & VADDEPALLY & Black & 76 & 143 \\
\hline & & Mean $=$ & $75.71 \%$ & 145 \\
\hline
\end{tabular}

Table 2 : The presence of Glomus species in Setaria italica from 8 mandals of 34 villages in Mahabubnagar district.

\begin{tabular}{|c|l|c|c|c|c|c|c|c|c|c|c|c|c|c|c|c|c|c|c|c|c|c|c|c|}
\hline $\begin{array}{c}\text { SIN } \\
\mathbf{0}\end{array}$ & $\begin{array}{l}\text { Name of the } \\
\text { village }\end{array}$ & $\mathbf{A}$ & $\mathbf{B}$ & $\mathbf{C}$ & $\mathbf{D}$ & $\mathbf{E}$ & $\mathbf{F}$ & $\mathbf{G}$ & $\mathbf{H}$ & $\mathbf{I}$ & $\mathbf{J}$ & $\mathbf{K}$ & $\mathbf{L}$ & $\mathbf{M}$ & $\mathbf{N}$ & $\mathbf{O}$ & $\mathbf{P}$ & $\mathbf{Q}$ & $\mathbf{R}$ & $\mathbf{S}$ & $\mathbf{T}$ & $\mathbf{U}$ & $\mathbf{V}$ & $\mathbf{W}$ \\
\hline $\mathbf{1}$ & Alampur & + & - & + & - & - & - & + & - & - & - & - & + & - & + & - & - & - & - & + & - & + & + & - \\
\hline $\mathbf{2}$ & Bheemavaram & + & - & - & - & - & - & + & + & - & - & - & + & - & - & - & - & + & + & - & - & - & - & - \\
\hline $\mathbf{3}$ & Koneru & - & + & + & + & - & - & - & - & - & + & - & + & - & + & - & + & - & - & - & + & - & - & + \\
\hline $\mathbf{4}$ & Kyatur & + & - & + & + & - & - & - & - & - & - & - & + & - & - & - & - & - & - & - & - & - & - & - \\
\hline $\mathbf{5}$ & Liganvai & - & - & - & + & - & - & - & + & + & - & + & + & + & - & - & - & - & - & - & - & - & - & - \\
\hline $\mathbf{6}$ & Maramunagala & - & + & - & - & + & - & + & - & - & - & - & + & - & - & + & - & - & - & - & + & - & - & - \\
\hline $\mathbf{7}$ & Utkur & - & + & - & - & - & - & - & - & - & - & - & + & - & - & - & - & - & + & - & - & - & - & - \\
\hline $\mathbf{8}$ & Ettam & - & - & + & - & - & - & - & - & - & - & - & + & - & - & - & - & + & - & - & - & - & - & - \\
\hline $\mathbf{9}$ & Narsaipalle & - & - & + & - & - & - & - & - & - & - & - & + & - & - & - & - & + & + & - & - & - & + & - \\
\hline $\mathbf{1 0}$ & Chukkaipalle & - & - & - & - & - & + & - & - & - & - & - & + & - & + & + & + & - & - & - & - & - & - & - \\
\hline $\mathbf{1 1}$ & Kollapur & - & - & - & - & - & - & - & - & - & + & - & + & - & - & - & - & - & - & - & + & - & - & - \\
\hline $\mathbf{1 2}$ & Kudikilla & - & - & - & - & - & - & + & - & - & - & - & + & - & - & - & - & - & + & - & - & + & - & - \\
\hline & Madhava Swamy & - & - & + & - & - & - & - & - & - & + & - & + & - & - & - & - & + & - & - & - & - & - & + \\
$\mathbf{1 3}$ & Nagar & & & & & & & & & & & & & & & & & & & & & & & \\
\hline $\mathbf{1 4}$ & Malleswaram & - & - & - & + & - & - & + & - & - & - & - & + & - & - & - & - & + & - & - & - & - & - & - \\
\hline $\mathbf{1 5}$ & Manchalakatta & - & - & + & - & - & - & - & - & - & - & - & + & - & - & - & - & - & - & + & - & - & - & - \\
\hline $\mathbf{1 6}$ & Pentlavelly & - & - & - & - & - & - & - & - & - & + & - & + & - & - & - & + & - & - & - & - & - & - & - \\
\hline $\mathbf{1 7}$ & Ramapur & - & - & - & - & - & + & - & - & - & - & - & + & - & - & - & - & + & - & - & - & - & - & - \\
\hline
\end{tabular}




\begin{tabular}{|c|c|c|c|c|c|c|c|c|c|c|c|c|c|c|c|c|c|c|c|c|c|c|c|c|}
\hline 18 & Somashila & - & - & - & + & - & - & - & - & - & - & - & + & - & - & - & 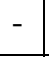 & - & - & - & + & - & - & - \\
\hline 19 & Yangam pally & - & - & - & - & - & - & - & - & - & - & - & + & - & - & + & + & - & + & - & - & - & - & - \\
\hline 20 & Yellur & - & - & + & - & - & + & - & - & + & - & - & + & + & - & - & - & - & - & - & - & - & - & - \\
\hline 21 & Ieeja & - & + & - & - & - & - & + & - & - & - & - & + & - & - & - & - & - & - & - & - & - & + & - \\
\hline 22 & Tuppaditalla & - & - & - & - & - & - & - & - & - & + & - & + & - & + & - & - & - & - & + & - & + & - & - \\
\hline 23 & Venkatapur & - & - & - & - & - & - & - & - & - & - & - & + & - & - & + & + & - & - & - & - & - & - & - \\
\hline 24 & Yapadinne & - & - & + & - & - & - & - & - & - & - & - & + & - & - & - & - & + & - & - & - & + & - & - \\
\hline 25 & Itikyal & + & - & - & - & + & - & - & + & - & + & - & + & - & - & - & - & - & + & - & - & - & - & - \\
\hline 26 & Saterla & - & - & - & + & - & + & - & - & - & - & - & + & - & + & + & - & - & - & - & + & - & - & - \\
\hline 27 & Udandapur & - & - & - & - & - & - & - & - & - & + & - & + & - & - & + & - & - & - & - & - & - & - & - \\
\hline 28 & Kalugotla & + & - & - & - & + & + & - & - & - & - & - & + & - & - & - & - & - & - & - & + & - & - & - \\
\hline 29 & Potulapadu & - & + & + & - & - & - & + & - & - & - & - & + & - & - & - & - & + & - & - & - & - & - & - \\
\hline 30 & Konduru & - & + & - & - & + & - & - & - & + & - & - & + & + & - & + & - & - & - & - & - & - & - & - \\
\hline 31 & Rajoli & - & - & - & - & - & - & - & + & + & - & - & + & - & - & - & - & + & - & - & - & - & - & - \\
\hline 32 & Ramapuram & - & - & - & - & - & - & - & - & - & + & - & + & - & - & - & - & + & - & - & - & + & - & - \\
\hline 33 & Timmajipalle & - & + & - & - & + & - & - & - & + & - & - & + & - & - & - & + & - & - & + & - & - & - & - \\
\hline 34 & Vaddepally & - & - & + & - & - & - & - & - & - & - & + & + & - & - & + & - & - & - & - & - & - & - & + \\
\hline
\end{tabular}

$\mathrm{A}=$ G.aggregatum $\mathrm{B}=$ G.arborense, $\mathrm{C}=$ G.aureum $; \mathrm{D}=$ G.badium $; \mathrm{E}=$ G.citricola; $\mathrm{F}=$ G.claroidium; $\mathrm{G}=$ G. clarum; $\mathrm{H}=$ G. constrictum; $\mathrm{I}=$ G. cubense;

$J=G$.dimorphicum; $K=G$.etunicatum; $L=G$.fasciculatum; $M=G$.globiferum; $N=G$.glomerulatum; $O=G$.la mellosum; $P=G$.macrocarpum;

$Q=G$.maculosum; $R=G$.microaggregatum; $S=G$.mosseae; $T=G$.tenebrosum; $U=G$. versiforme; $V=G$.visco sum; $W=G$. warcu

\section{Cite this article as :}

Hari Prasad Kante, Laxmi Kanth Mhadgula, "Glomus fasciculatum, a dominant arbuscular mycorrhizal species in the rhizosphere soils of Setaria italica in Mahabubnagar district of Telangana state, India", International Journal of Scientific Research in Science and Technology (IJSRST), Online ISSN : 2395-602X, Print ISSN : 2395-6011, Volume 6 Issue 6, pp. 165-170, November-December 2019. Available at doi : https://doi.org/10.32628/IJSRST196629 Journal URL : http://ijsrst.com/IJSRST196629 